\title{
The Good and the Bad: Environmental Efficiency in Northeastern U.S. Dairy Farming
}

\section{Eric Njuki, Boris E. Bravo-Ureta, and Deep Mukherjee}

\begin{abstract}
This study evaluates the environmental performance of northeastern U.S. dairy operations that differ in size using a directional output-distance function that measures the joint production of milk and emissions while incorporating a fourway error approach that captures farm-size heterogeneity, transient and persistent technical efficiency, and random errors. For the emission component, a comprehensive pollution index is generated that incorporates three major sources of pollution in dairy farming: fuel, fertilizer, and livestock. Computed shadow prices and Morishima elasticities of substitution reveal that large dairy operations are environmentally inefficient compared to their smaller counterparts.
\end{abstract}

Key Words: dairy farming, directional output-distance function, emissions, environmental efficiency, shadow prices, undesirable output

Dairy farming is the largest agricultural sector in the northeast United States ${ }^{1}$ (Winsten et al. 2010). It contributes to the local economy through direct generation of producer income, purchase of inputs and services from local providers, and employment. According to the National Agricultural Statistics Service (NASS), dairy operations in the Northeast in 2013 generated 17.5 billion pounds of milk and 3.7 billion dollars in household income (U.S. Department of Agriculture (USDA) 2014). Moreover, as the Northeast has become increasingly urbanized, the importance of dairy farms in preserving rural landscapes and open spaces has been brought to the forefront (Johnston 2002). These ecosystem services are responsible for maintaining heritage values, rural vitality, and the ambience of the region (Batie 2003).

Benefits notwithstanding, the dairy sector in the Northeast faces serious challenges. Most notably, economies of scale and rapidly changing technologies are

Eric Njuki is a USDA NIFA postdoctoral fellow and Boris E. Bravo-Ureta is a professor in the Department of Agricultural and Resource Economics at University of Connecticut. Deep Mukherjee is an assistant professor at the India Institute of Technology, Kanpur, India. Correspondence: Eric Njuki - 1376 Storrs Road, Unit 4021 - Storrs, CT 06269 - Phone 860.486.2917 - Email eric. njuki@uconn.edu.

The authors gratefully acknowledge the financial support of U.S. Department of Agriculture, National Institute of Food and Agriculture grant 2011-67024-30912, and the Zwick Center for Food and Resource Policy at the University of Connecticut. The views expressed are the authors' and do not necessarily represent the policies or views of the sponsoring agency.

1 The states comprising the northeast United States in this study are New York, Connecticut, Maine, Massachusetts, New Hampshire, Rhode Island, and Vermont.

Agricultural and Resource Economics Review 45/1 (April 2016) 22-43

(C) The Author(s) 2016. This is an Open Access article, distributed under the terms of the Creative Commons Attribution licence (http://creativecommons.org/licenses/by/4.0/), which permits unrestricted re-use, distribution, and reproduction in any medium, provided the original work is 


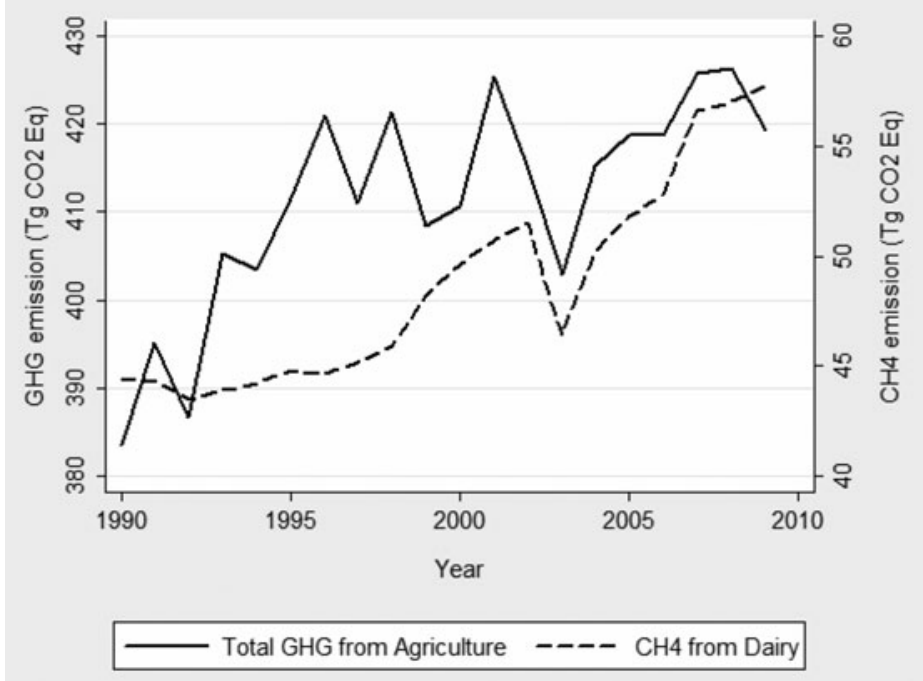

Figure 1. Trend in GHG Emissions from the U.S. Agricultural Sector Source: U.S. EPA (2011).

driving dairies to consolidate into larger operations at the expense of smaller familyoperated farms. A national trend of fewer larger dairy farms has been observed for a number of years (MacDonald, McBride, and O'Donoghue 2007); according to NASS (USDA 2010), the total number of dairy operations nationwide had decreased 83 percent since 1960. In addition, environmental problems created by pollution have raised concern about the industry's environmental sustainability (Thoma et al. 2013). Dairy farms are responsible for generating pollutants such as carbon dioxide, methane, nitrogen oxide, ammonia, and acid gases (Environmental Protection Agency (EPA) 2012a). The greenhouse gas (GHG) emissions originating from dairy operations have been on an upward trend (EPA 2012a), accounting for 26 percent of total methane and 12 percent of total GHGs generated by the U.S. agricultural sector from 1990 through 2009 (EPA 2011). Figure 1 illustrates the evolution of methane emissions emanating from U.S. dairy farms.

Quantifying the environmental impact of GHGs poses various challenges. A key issue is that these GHGs, hereafter referred to as emissions, are not priced in markets, making it difficult to derive monetary measures of their environmental impacts. Moreover, these emissions have been termed "uniformly mixed assimilative pollutants" because they disperse from their point source of pollution over a large area and accumulate over time when the discharge rate exceeds the absorptive capacity of the environment (Tietenberg 2006). A consequence of these characteristics is that the sources of such pollution are difficult to inventory.

This study measures and analyzes environmental efficiency for groups of northeastern U.S. dairy operations that differ in size. Environmental efficiency analysis evaluates the tradeoff between production of a desirable good and 
associated emissions (Fernandez, Koop, and Steel 2002). In this study, the first step in the analysis is construction of an emission index that incorporates the three major types of pollution emanating from dairy operations-livestock, fertilizer, and fuel-following Njuki and Bravo-Ureta (2015). Thereafter, the joint production of the desirable output (dairy products) and emissions is analyzed to estimate the cost to society of those emissions as well as to establish whether environmental efficiency has improved or deteriorated over time.

\section{Environmental Efficiency and Polluting Technologies}

Over the years, productivity analyses have focused mainly on two major components, technical efficiency and technological change, while ignoring environmental efficiency. More recently, the interest in environmental efficiency has increased as evidenced by a number of studies (e.g., Chung, Färe, and Grosskopf 1997, Färe et al. 2005, Atkinson and Dorfman 2005, O’Donnell 2007, Cuesta, Lovell, and Zofio 2009). So far, however, work related to environmental efficiency in dairy farming has been limited; notable examples are Reinhard, Lovell, and Thijssen (1999), Fernandez, Koop, and Steel (2002), Njuki and Bravo-Ureta (2015), and Qi, Bravo-Ureta, and Cabrera (2015).

This study builds on recent developments in productivity analysis that have examined both desirable outputs and emissions (bad outputs) generated by a productive unit. This approach differs from previous work related to dairy farming in its construction and modeling of the pollution index. Whereas the environmental effects in studies by Reinhard, Lovell, and Thijssen (1999) and Fernandez, Koop, and Steel (2002) were based only on the nitrogen surplus from fertilizer applications, the emission index constructed in this study also includes methane and nitrous oxide emissions from livestock and carbon dioxide emissions from fuel. According to Gerber et al. (2013), feed production and processing are responsible for at least 45 percent of the GHGs emanating from livestock operations. Digestion by cows accounts for another 39 percent and manure decomposition accounts for 10 percent. The remainder of the emissions can be attributed to processing and transportation of animal products. Therefore, the emission index constructed in this study captures a sizable share of dairy operation emissions.

Researchers and stakeholders in the dairy sector became concerned about environmental efficiency in the Northeast because the EPA (2009) declared that GHGs posed a threat to public health and welfare and would be regulated under the Clean Air Act Amendment (1990), ${ }^{2}$ which was meant to control three major threats to the environment: acid rain, urban pollution, and toxic air emissions. Specifically, EPA sought to control those environmental threats using economic incentives in a market-driven process that included performance-based

2 This declaration followed Commonwealth of Massachusetts et al. vs. EPA et al., a lawsuit decided by the Supreme Court in 2007. 
standards and banking and trading of emissions. In its 2009 report, "Mandatory Reporting of Greenhouse Gases; Final Rule," EPA provided a set of guidelines that would impose strict standards for reporting of GHG emissions in several sectors of the U.S. economy, including the livestock sector. Since implementation of the policy could have far-reaching implications, this analysis measures the impacts of emissions on the environmental efficiency of dairy farm operations in the northeastern United States.

\section{Methodology}

The theoretical foundation for modeling a multi-output technology is Shephard's (1970) output-distance function (ODF). However, this function is not appropriate for modeling polluting technologies because it radially expands the desirable output and the emissions toward the frontier. Its proportional expansion of all outputs, good and bad, is not compatible with the goal of minimizing pollution.

An alternative to Shephard's ODF is the directional distance function developed by Chambers, Chung, and Färe (1996) and later extended as a technique for modeling polluting technologies by Chung, Färe, and Grosskopf (1997). Allowing the directional vectors for both inputs and outputs to vary generates a directional technology-distance function (Färe 2010). In this study, the assumed technology follows previous studies that restricted the directional input vector to zero (e.g., Chung, Färe, and Grosskopf 1997, Färe et al. 2005, O'Donnell 2007). Hence, it is a directional output-distance function (DODF) that relies on two key assumptions: (i) in a multi-dimensional production frontier, the decision-making unit's (DMU's) objective is to simultaneously expand production of the desirable output and contract production of emissions; (ii) the directional vector can follow numerous projections to the frontier of the output set. The distance between the frontier and the observed output in the direction that reduces emissions while not decreasing the desirable output is the firm's environmental technical efficiency.

We begin by defining a technology as a technique, method, or process for transforming inputs into outputs. In addition, there are environmental factors, $z \in \mathfrak{R}_{+}^{j}$, that are exogenous and involved in the production process but are beyond the control of the DMU. The set of feasible combinations of inputs and outputs includes a vector of $k$ inputs, $x \in \mathfrak{R}_{+}^{k}$, and vectors representing the desirable output and emissions, $y \in \mathfrak{R}_{+}^{m}$ and $b \in \mathfrak{R}_{+}^{i}$, respectively. Therefore, the environment-specific technology set can be defined (see O'Donnell 2016) as

$$
\begin{aligned}
T(z)= & \left\{(x, y, b): x \in \mathfrak{R}_{+}^{k}, y \in \mathfrak{R}_{+}^{m},\right. \\
& \left.b \in \mathfrak{R}_{+}^{i}: x \text { can produce }(y, b) \text { in environment } z\right\} .
\end{aligned}
$$

We also assume an output set, $P(x, z)=\{(y, b):(x, y, b) \in T(z)\}$, that is defined to be 
a multi-dimensional production-possibility frontier that represents the combination of production of the desirable output and of emissions $(y, b)$ generated by the DMU using the input vector $\mathbf{x}$ in environment $\mathbf{z}$. The output set satisfies standard production axioms (see Färe and Primont 1995). In addition, we assume null-jointness - the good and the bad are produced jointly. Thus, if output of $\mathbf{b}$ is zero, it is not possible to generate any of good $\mathbf{y}$ (Shephard and Färe 1974). Following Shephard (1970), we further assume that the outputs are weakly disposable such that any proportional amounts of contraction of the good and of the bad output are feasible. The weak disposability property implies that disposing of emissions is costly because some inputs must be redirected from producing the good output to emission reduction; that is, abatement requires a reduction in the firm's activity level (Kuosmanen 2005).

When $g=\left(g_{y},-g_{b}\right)$ represents the directional vector, the environment-specific DODF is defined as

$$
\vec{D} o\left(\mathbf{x}, z, \mathbf{y}, \mathbf{b} ; g_{y},-g_{b}\right)=\max \left\{\beta:\left(y+\beta g_{y}, \mathbf{b}-\beta g_{b}\right) \in P(x, z)\right\}
$$

where $\mathbf{x}, \mathbf{y}$, and $\mathbf{b}$ are vectors of the inputs, the desirable output, and emissions respectively and $\mathbf{z}$ represents the exogenous environmental factors as already noted. We define $\beta$ as a scaling factor, and the firm's objective is to expand production of the good output by $\beta g_{y}$ while contracting emissions by $\beta g_{b}$. The directional vector is exogenously determined and hence can take a variety of values.

The properties of the DODF are inherited from the output set and can be specified as follows. First, the DODF is nonnegative for all feasible output vectors $(y, b) \in P(x, z)$. Second, it is concave in $(y, b) \in P(x, z)$. Third, it exhibits monotonicity, which corresponds to strong disposability of desirable outputs. This property is denoted as

$$
\begin{aligned}
\vec{D} o\left(x, z, y^{\prime}, b ; g_{y},-g_{b}\right) & \geq \vec{D} o\left(x, z, y, b ; g_{y},-g_{b}\right) \text { for }\left(y^{\prime}, b\right) \leq(y, b) \\
& \in P(x, z) .
\end{aligned}
$$

According to equation 3, inefficiency does not increase if a firm, using the same amount of inputs, generates more of the good output and the same amount of the bad output. Under the fourth property, when the firm's production of emissions rises while production of the desirable output and of inputs remains constant, inefficiency does not decrease; that is (Färe et al. 2005),

$$
\begin{aligned}
\vec{D} o\left(x, z, y, b^{\prime} ; g_{y},-g_{b}\right) & \geq \vec{D} o\left(x, z, y, b ; g_{y},-g_{b}\right) \text { for }\left(y, b^{\prime}\right) \leq(y, b) \\
& \in P(x, z) .
\end{aligned}
$$

The fifth property of the DODF is weak disposability for both goods, which can be expressed as 


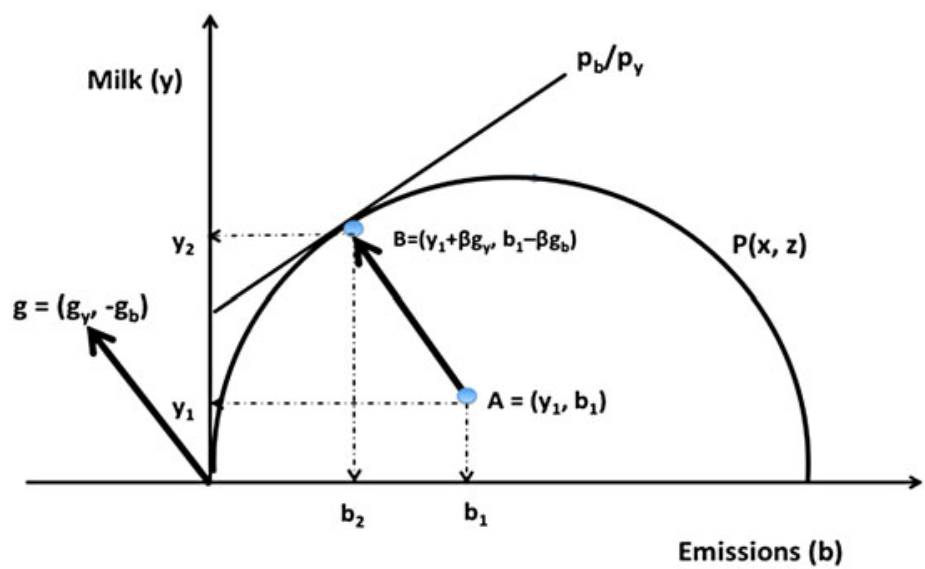

Figure 2. Directional Output Distance Function

$$
\vec{D} o\left(x, z, \theta y, \theta b ; g_{y},-g_{b}\right) \geq 0 \text { for }(y, b) \in P(x, z) \text { and } 0 \leq \theta \leq 1 \text {. }
$$

This equation indicates that a reduction in emissions requires a decrease in the firm's activity level (Kuosmanen 2005). Finally, the sixth property is translation, which is analogous to homogeneity of degree one in Shephard's (1970) outputdistance function. The translation property is expressed as

$$
\begin{aligned}
\vec{D} o\left(x, z, y+\beta g_{y}, b-\beta g_{b} ; g_{y},-g_{b}\right)= & \vec{D} o\left(x, z, y, b ; g_{y},-g_{b}\right)-\beta, \\
& \text { for } \beta \in \mathfrak{R} .
\end{aligned}
$$

According to the translation property, the value of the resulting distance function will be more efficient by $\beta$ if the vector of the good output expands by scaling factor $\beta g_{y}$ and the bad output contracts by $\beta g_{b}$ (Färe et al. 2005).

Figure 2 provides a graphical illustration of the DODF. The representative firm in this illustration initially produces inside the output set, $P(x, z)$, at point $A=\left(y_{1}, b_{1}\right)$. The objective is to increase the firm's efficiency by simultaneously expanding production of the desirable output and contracting production of emissions. This can be accomplished by scaling the vector from point $A=\left(y_{1}, b_{1}\right)$ to point $B=\left(y_{1}+\beta g_{y}, b_{1}-\beta g_{b}\right)$. The efficient combination of the desirable output and emission levels is determined by the tangency between the price ratio $\left(p_{b} / p_{y}\right)$ and the frontier of the output set, $P(x, z)$, at point B. Since emissions are not priced in markets, tangency of the price ratio and the frontier allows us to estimate their shadow price, $p_{b}$. The vector $g=\left(g_{y},-g_{b}\right)$ represents the directional vector, which is determined exogenously following common practice (e.g., 
Chung, Färe, and Grosskopf 1997, Färe et al. 2005). By the translation property, the scaling of the vector from point A to point B parallel to the directional vector toward the frontier represents a solution to

$$
\vec{D} o\left(x, z, y, b ; g_{y},-g_{b}\right)=\max \left\{\beta:\left(y+\beta g_{y}, b-g_{b}\right) \in P(x, z)\right\}
$$

At the point of tangency, the representative firm has fully improved its environmental technical efficiency (ETE).

\section{Empirical Specification}

The parametric stochastic frontier takes the form

$$
\vec{D} o(x, z, y, b ; 1,-1)+\left(w_{i}+\varepsilon_{\mathrm{it}}\right)=0 .
$$

The last term represents a two-part disturbance that captures the unobserved firm effects, time-invariant persistent inefficiency, time-varying transient inefficiency, and random errors (the error specification is discussed in more detail hereafter).

A quadratic DODF is implemented while imposing values of $g=(1,-1)$ on the directional vector, which represent equal weights assigned to the desirable output and desirable emissions. In addition, this choice of directional vector emphasizes the weak disposability property of the DODF previously discussed. That is, instead of scaling the directional vector from point A to the highest point of the output set (see Figure 2), a reduction in emissions requires a decrease in the firm's activity level. Moreover, the directional vector provides analytical simplicity and convenience in interpreting the results and is consistent with the related literature (e.g., Chung, Färe, and Grosskopf 1997, Färe et al. 2005, PicazoTadeo, Reig-Martinez, and Hernandez-Sancho 2005, Njuki and Bravo-Ureta 2015).

The model can be written as

$$
\begin{aligned}
\vec{D} o(x, z, y, b ; 1,-1)= & \alpha 0+\sum_{n=1}^{4} \alpha_{n} x_{n i t}+\sum_{n=1}^{2} \delta_{n} z_{n t} \\
& +\pi_{1} t+\pi_{2} t^{2}+\phi_{1} y_{i t}+\gamma_{1} b_{i t} \\
& +\frac{1}{2} \phi_{2} y_{1 i t}^{2}+\frac{1}{2} \gamma_{2} b_{i t}^{2}+\left(\omega_{i}+\varepsilon_{i t}\right) .
\end{aligned}
$$

The variable $\mathbf{x}_{n i t}$ represents conventional inputs: cows, capital, labor, and purchased feed respectively. Intermediate materials used in the production process are embedded in capital. The variable $\mathbf{z}_{n t}$ captures 30-year moving averages of two climate variables, temperature and precipitation. The variables $t$ and $t^{2}$ represent a linear and a quadratic time trend that capture 
technical change in the model; ${ }^{3}$ and, finally, $\mathbf{y}_{i t}$ and $\mathbf{b}_{i t}$ measure milk output and emissions respectively. For the translation property to hold and to account for the choice of the directional vector, we impose the parametric restriction $\varphi_{1}$ $\gamma_{1}=-1$ (Färe et al. 2005).

Recent studies have developed a four-way error structure to disentangle unobserved firm effects, time-invariant persistent technical efficiency, timevarying transient technical efficiency, and random errors (e.g., Colombi et al. 2014, Filippini and Greene 2014, Kumbhakar, Lien, and Hardaker 2014, Tsionas and Kumbhakar 2014). In the dairy sector, persistent inefficiency levels can arise from institutional and statutory regimes such as minimum pricing laws and food and safety regulations. Transient inefficiency levels, on the other hand, are derived from shocks associated with implementing new technologies (Kumbhakar, Tsionas, and Sipilainen 2009) and human capital (Kumbhakar, Ghosh, and McGuckin 1991). Furthermore, unobserved firm effects come about because firms adjust their use of inputs in response to constantly changing production environments, a process that is observed by the producer but not by the econometrician (Kellerman 2015).

Following Filippini and Greene (2014), we employ a generalized true random effects (GTRE) approach that nests the pooled frontier and true random effects models. Consequently, the error structure in equation 7 $\left(\omega_{i}+\varepsilon_{i t}\right)$ is a two-part disturbance. The first part, $\omega_{i}=\xi_{i}-\eta_{i}$, is a timeinvariant component in which $\xi_{i}$ captures firm heterogeneity and $\eta_{i}$ captures persistent technical inefficiency. This component follows a skew-normal distribution and has distributional parameters of $\kappa=\sigma_{\eta} / \sigma_{\xi}$ and $\theta=\left(\sigma_{\eta}^{2}+\right.$ $\left.\sigma_{\xi}^{2}\right)^{1 / 2}$. The second part, $\varepsilon_{i t}=v_{i t}-u_{i t}$, is a time-varying component. The term $\mathbf{v}_{i t}$ represents statistical error that captures measurement errors and functional form errors, and $\mathbf{u}_{i t}$ represents transient technical inefficiency. This component also follows a skew-normal distribution with parameters $\lambda=\sigma_{u} / \sigma_{v}$ and $\rho=\left(\sigma_{v}^{2}+\sigma_{u}^{2}\right)^{1 / 2}$.

As in Färe et al. (2005), we assume that, for the ith observation, the scaling factor $\beta^{i}$ is added to $\mathrm{y}^{i}$ and subtracted from $b^{i}$. We set $\beta^{i}=b^{i}$. Thus, we obtain variation on the lefthand side by choosing a specific $\beta^{i}$ for each observation. The functional form given in equation 7 can now be rewritten as

$$
\begin{aligned}
-(\text { emissions }= & \alpha_{0}+\alpha_{1} \text { cows }_{i t}+\alpha_{2} \text { capital }_{i t}+\alpha_{3} \text { labor }_{i t}+\alpha_{4} \text { feed }_{i t} \\
& +\delta_{1} \text { temp }_{t}+\delta_{2} \text { prec }_{t}+\pi_{1} \text { time }_{t}+1 / 2 \pi_{2} \text { time }_{t}^{2}+\varphi_{1} \text { milk }_{i t} \\
& +1 / 2 \varphi_{2} \text { milk }_{i t}^{2}+1 / 2 \gamma_{2} \text { emissions }_{i t}^{2}+\left(\omega_{i}+\varepsilon_{i t}\right) .
\end{aligned}
$$

3 Technical change is assumed to be Hicks-neutral; this corresponds to a homothetic shift in isoquants following Solow (1957). 
Shadow Prices and Morishima Elasticities of Output Substitution

The shadow price is defined as the dollar value of the emission output generated at the tangency of the price line $\left(p_{b} / p_{y}\right)$ and the output frontier $\left(P_{x}\right)$. The shadow price also can be defined as the marginal abatement cost and can be calculated by setting up the revenue function following Chambers, Chung, and Färe (1998):

$$
R\left(p_{y}, p_{b}\right)=\max _{y, b}\left\{p_{y} \cdot p_{b} \cdot b: \vec{D} o\left(x, z, y, b ; g_{y},-g_{b}\right)\right\} \geq 0
$$

where $p_{y}$ is the price of the desirable output, $p_{b}$ is the price of the emissions, and $\vec{D} o\left(x, z, y, b ; g_{y},-g_{b}\right)$ is the directional output-distance function. The Lagrangian expression associated with maximization of revenue subject to the DODF is

$$
\mathcal{L}=\max _{y, b}\left\{p_{y} \cdot y-p_{b} \cdot b+\lambda\left(0-\vec{D} o\left(x, z, y, b ; g_{y},-g_{b}\right)\right)\right\}
$$

To solve for the revenue function, we need to establish the value of $\lambda$. By making use of the translation property, the revenue function can be rewritten as

$$
\begin{aligned}
R\left(p_{y}, p_{b} ; \beta\right)= & \max _{y, b}\left\{p_{y}\left(y+\beta g_{y}\right)-p_{b}\left(b+\beta g_{b}\right):\right. \\
& \left.\vec{D} o\left(x, z, y,+\beta g_{y}, b+\beta g_{b} ; g_{y},-g_{b}\right) \geq 0\right\}+\beta\left(p_{y} g_{y}+p_{b} g_{b}\right) .
\end{aligned}
$$

The short form of this revenue function is

$$
R\left(p_{y}, p_{b} ; \beta\right)=R\left(p_{y}, p_{b}\right)-\beta\left(p_{y} g_{y}+p_{b} g_{b}\right)
$$

which implies that

$$
\partial R\left(p_{y}, p_{b} ; \beta\right) / \partial \beta=-\left(p_{y} g_{y}+p_{b} g_{b}\right) .
$$

By incorporating the translation property into the Lagrangian expression, we obtain

$$
\mathcal{L}=\max _{y, b}\left\{p_{y} \cdot y-p_{b} \cdot b+\lambda\left(\beta-\vec{D} o\left(x, z, y, b ; g_{y},-g_{b}\right)\right\} .\right.
$$

Then, we apply the envelope theorem to arrive at 


$$
\begin{aligned}
\partial \mathcal{L} / \partial \beta & =\partial R / \partial \beta=\partial / \partial \beta\left\{p_{y} \cdot y-p_{b} \cdot b+\lambda\left(\beta-\vec{D} o\left(x, z, y, b ; g_{y},-g_{b}\right)\right\}\right. \\
& =\lambda
\end{aligned}
$$

Using the initial values for the directional vector $(1,-1)$, we solve for the revenue function:

$$
\begin{aligned}
R\left(p_{y}, p_{b} ; 1,-1\right)= & \max _{y, b}\left\{p_{y} \cdot y-p_{b} \cdot b:\left(p_{y} \cdot 1+p_{b} \cdot 1\right)\right. \\
& \times \vec{D} o(x, z, y, b ; 1,-1)\} .
\end{aligned}
$$

The first-order conditions associated with the revenue function are

$$
\left(p_{y} \cdot g_{y}-p_{b} g_{b}\right) \nabla_{y} \vec{D} o\left(x, z, y, b ; g_{y},-g_{b}\right)=-p_{y}
$$

and

$$
\left(p_{y} \cdot g_{y}-p_{b} g_{b}\right) \nabla_{b} \vec{D} o\left(x, z, y, b ; g_{y},-g_{b}\right)=p_{b}
$$

The ratio from those expressions gives the relative shadow price as

$$
p_{y} / p_{b}=\left\{\nabla_{y} \vec{D} o\left(x, z, y, b ; g_{y},-g_{b}\right)\right\} /\left\{\nabla_{b} \vec{D} o\left(x, z, y, b ; g_{y},-g_{b}\right)\right\}
$$

We turn next to the Morishima elasticity of substitution (MES), which Blackorby and Russell (1989, p. 883) defined as "a measure of curvature, or ease of substitution." More recently, Färe et al. (2005) defined the MES as a measure of the change in the ratio of the price of the desirable output to the price of the emissions relative to a change in the quantity of the desirable output and emissions:

$$
M E S_{b y}=\left\{\partial \ln \left(p_{b} / p_{y}\right) / \partial \ln (y / b)\right\}
$$

In our case, the MES is an indicator of the ability of the dairy operation to trade reductions in milk for reductions in emissions. Based on the DODF used in our model, the MES can be specified as 


$$
\begin{aligned}
M E S_{b y}= & y\left[\frac{\partial^{2} \vec{D} o(x, z, y, b ; 1,-1) / \partial b^{2}}{\partial \vec{D} o(x, z, y, b ; 1,-1) / \partial b}\right. \\
& \left.-\frac{\partial^{2} \vec{D} o(x, z, y, b ; 1,-1) / \partial y^{2}}{\partial \vec{D} o(x, z, y, b ; 1,-1) / \partial y}\right]
\end{aligned}
$$

\section{Data}

The data set used in this analysis is derived from annual publications prepared by Farm Credit East (FCE), a financial institution and specialized lender that caters to farm businesses in the Northeast. These annual publications assess the financial health and progress of dairy farm businesses. Our data set covers dairy operations located in Connecticut, Maine, Massachusetts, New Hampshire, Rhode Island, Vermont, and New York and spans the 34-year period of 1980 through 2013. The dairies covered in the reports received most of their incomes from milk sales; between 2009 and 2013, for example, milk sales represented 83.2-88.3 percent of the aggregate annual income received by the dairies.

To preserve confidentiality, the FCE does not make individual farm-level data available. Instead, the data are aggregated and averaged at the farm level and reported on an annual basis for four categories of herd size. Hence, the units of observation in this study correspond to those categories. For the 34-year period covered by the data, we obtained 136 observations (dairies) that we grouped into small dairies, which had fewer than 90 cows; medium dairies, which had 90 to 149 cows; large dairies, which had 150 to 299 cows; and very large dairies, which had 300 or more cows. The number of dairies that participated in the annual survey ranged from 391 in 1980 to 789 in 1992.

Descriptive statistics for the variables in the data set are outlined in Table 1. The quantity of feed purchased was constructed by dividing nominal figures for total feed expense per cow by the nominal price for a 16-percent feed concentrate per year using prices obtained from the New England regional office of NASS. The variable for labor, expressed in worker-equivalent-hours, was collected and summarized by the FCE. All monetary values were converted to constant 2013-dollar values using the producer price index derived from the Bureau of Labor Statistics.

Next, the data were augmented with estimates of emissions using a comprehensive pollution index and climate variables that consisted of 30year moving averages of daily regional temperature and precipitation derived from the National Oceanic and Atmospheric Administration. These were included in the model in response to evidence that variations in climate affect dairy production activities (e.g., Mukherjee, Bravo-Ureta, and De Vries 2013, Key and Sneeringer 2014, Njuki and Bravo-Ureta 2015, Qi, Bravo-Ureta, and Cabrera 2015). The large size of the numerical values of the outputs and inputs resulted in convergence difficulties; thus, following Färe et al. (2005), 
Table 1. Descriptive Statistics

\begin{tabular}{lrrrr}
\hline Variable & Mean & Std. Dev. & Min. & \multicolumn{1}{c}{ Max. } \\
\hline Emissions - tons & $1,156.7$ & $1,203.8$ & 470.0 & $7,261.0$ \\
Milk - tons & $1,736.2$ & $2,127.4$ & $5,610.7$ & $12,612.8$ \\
Cows - number & 186.6 & 192.8 & $6,566.5$ & $1,110.0$ \\
Labor - hours & 4.5 & 3.6 & 1.0 & 21.4 \\
Capital - dollars & $100,701.0$ & $86,357.0$ & 0.5 & $523,773.0$ \\
Purchased feed - tons & 956.6 & $1,067.3$ & 0.1 & 4859.4 \\
Temperature - degrees Fahrenheit & 44.4 & 0.5 & 43.9 & 45.5 \\
Precipitation - inches & 3.7 & 0.1 & 3.6 & 3.9 \\
\hline
\end{tabular}

we normalized the data by dividing each output and input value by its mean value before estimating equation 8 .

\section{Construction of the Undesirable Output}

As previously noted, a recent report by Gerber et al. (2013) indicated that a significant share of GHGs emanating from livestock operations can be attributed to feed production and processing, digestion by cows, and manure decomposition. By constructing an index that captures emissions associated with livestock, fuel, and fertilizer, we account for a significant share of the emissions generated by the milk production portion of the fluid milk supply chain.

The index of emissions from fuel is constructed using data on each dairy's expenditures for gasoline and oil. Expenditures are converted to quantities of fuel consumed in gallons based on historical data on conventional gasoline prices from the U.S. Department of Energy's Energy Information Administration, and the equivalent emissions of carbon dioxide from those fuels are computed using the EPA's GHG equivalencies calculator (EPA 2012b).

The index of fertilizer-based emissions is constructed using information on the dairies' fertilizer expenditures and historical fertilizer prices from NASS to convert the expenditures to quantities. The amount of direct emission of nitrous oxide, which is derived from nitrogen applied to the soil via fertilizers, is calculated using formulations by Mosier (1994).

For the livestock-based emissions, we use methodologies outlined in EPA guidelines $(2009, \text { p. } 56481)^{4}$ that were designed to improve the accuracy of information collected regarding GHG emissions and to better understand the

\footnotetext{
4 Various stakeholders expressed specific concerns about these methodologies, and EPA addressed those concerns in its report (EPA 2009, p. 56264, 56338-56339).
} 
sources of those emissions. These guidelines were authorized by congress under the Consolidated Appropriations Act (2008). The livestock component of the emissions index is measured in metric tons of carbon dioxide equivalent, which accounts for methane and nitrous oxide generated by animals at dairy facilities. The amount of methane emitted is a product of total volatile solids (VS) excreted per animal (which varies with the type of animal), the fraction of the VS that is methane (also varies by animal type), and a conversion factor for the global warming potential of methane.

Total VS is a product of the average annual animal population at the facility, the typical mass of each animal type (for dairy cows, the default value is 604 kilograms (kg)), and the VS excretion rate for type of animal, which varies by state. In the northeast United States, these rates range from 8.63 VS per day per $1,000 \mathrm{~kg}$ of animal mass in Massachusetts to $9.44 \mathrm{VS}$ per day per 1,000 $\mathrm{kg}$ of animal mass in New Hampshire. We use the average for the region, 9.09. The resulting estimates of methane produced are then multiplied by 21 , the global warming potential of methane. We compute the amount of livestock-based nitrous oxide emissions using the same calculations. The daily nitrogen excretion rate for the northeastern United States ranges from $0.51 \mathrm{VS}$ per day per $1,000 \mathrm{~kg}$ of animal mass in Massachusetts to $0.54 \mathrm{~kg}$ of VS per day per $1,000 \mathrm{~kg}$ of animal mass in New Hampshire. We use the average for the region, 0.525 . The resulting estimates of nitrous oxide produced are then multiplied by the conversion factor of 310 to determine the potential for global warming.

The combination of these three major sources of pollution determines total emissions. Table 1 provides a summary of the variables used in the analysis.

\section{Results}

Table 2 presents the estimated coefficients, standard errors, and results of the distributional parameters: $\lambda, \rho, \sigma_{\eta}$, and $\sigma_{\xi}$. The estimated coefficients are then used to compute shadow prices and Morishima elasticities of substitution.

In terms of the distributional parameters, the ratio of $\sigma_{u}$ to $\sigma_{v}$ is denoted by $\lambda$. This ratio signifies the relative importance of the transient inefficiency term $u_{i t}$ with respect to the two-sided statistical error term $v_{i t}$. The estimated value of $\lambda$ is 1.94, which implies that the one-sided transient inefficiency component dominates any statistical error in determination of the time-varying component, $\varepsilon_{i t}$. The variance of the persistent inefficiency term $\sigma_{\eta}$, on the other hand, is 0.70 and dominates the variance of the farm-size heterogeneity term $\sigma_{\xi}, 0.03$. Hence, we conclude that the persistent inefficiency term largely determines the composition of the time-invariant component $\omega_{i}$.

Tables 3 and 4 report the estimates of transient and persistent technical efficiency by farm size. The kernel densities for the efficiency estimates are plotted in Figure 3. These estimates are higher on average than ones of technical efficiency from studies of the stochastic production frontier for U.S. 
Table 2. Estimated Random Coefficients from the Frontier Model

\begin{tabular}{lrrrr}
\hline Variable & Coefficient & Std. Error & \multicolumn{1}{c}{$\mathbf{Z}$} & $|\mathbf{z}|>\mathbf{Z}^{*}$ \\
\hline$\alpha_{1}$ & $0.7261^{*}$ & 0.1036 & 7.0100 & 0.0000 \\
$\alpha_{2}$ & $0.0635^{* *}$ & 0.0303 & 2.1000 & 0.0359 \\
$\alpha_{3}$ & $0.1549^{* *}$ & 0.0783 & 1.9800 & 0.0479 \\
$\alpha_{4}$ & $-0.1023^{*}$ & 0.0100 & -10.2000 & 0.0000 \\
$\delta_{1}$ & $8.7172^{*}$ & 1.0114 & 8.6200 & 0.0000 \\
$\delta_{2}$ & $2.0297^{*}$ & 0.2613 & 7.7700 & 0.0000 \\
$\pi_{1}$ & $-0.0392^{*}$ & 0.0266 & -1.4700 & 0.1403 \\
$\pi_{2}$ & $-0.1101^{*}$ & 0.0152 & -7.2700 & 0.0000 \\
$\phi_{1}$ & $0.1395^{*}$ & 0.0482 & 2.9000 & 0.0038 \\
$\phi_{2}$ & $-0.0954^{*}$ & 0.0024 & -39.8800 & 0.0000 \\
$\gamma_{2}$ & $0.1372^{*}$ & 0.0043 & 32.2800 & 0.0000 \\
$\alpha_{0}$ & $-10.5786^{*}$ & 0.9296 & -11.3800 & 0.0000 \\
$\lambda$ & $1.9400^{*}$ & 0.1638 & 11.8437 & 0.0000 \\
$\rho$ & $0.0647^{*}$ & 0.0037 & 17.6700 & 0.0000 \\
$\sigma_{\eta}$ & $0.7044^{*}$ & 0.0577 & 12.2100 & 0.0000 \\
$\sigma_{\xi}$ & $0.0270^{*}$ & 0.0017 & 16.0200 & 0.0000 \\
\hline
\end{tabular}

Log simulated likelihood: 205.063

Note: ${ }^{*}$ denotes significance at the 1 percent level, ${ }^{* *}$ at the 5 percent level, and ${ }^{* * *}$ at the 10 percent level.

dairies (e.g., Ahmad and Bravo-Ureta 1996, Bravo-Ureta et al. 2007). Moreover, farm size has no notable effect on efficiency.

Table 5 reports the estimated shadow prices. To illustrate the meaning of the shadow price in this context, consider the $\$ 215.4$ value for small farms in 1980 . This shadow price indicates that an increase in milk output at the margin would have generated an additional $\$ 215.4$ worth of emissions. Alternatively, this is the dollar value that the average small farm would have had to forfeit in 1980 to reduce emissions by one ton.

In terms of farm size, our results are mixed. The lowest estimated shadow price is $\$ 102.6$ for very large farms in 1981 . On the other hand, the highest estimated shadow price is $\$ 314.7$ in 2008 , again for very large farms. In general, at the beginning of the sample period, small farms consistently faced the highest shadow prices. However, beginning in the mid-1990s, the estimated shadow prices for very large farms began to exceed those of smaller farms. Thus, larger operations would have faced greater marginal abatement costs relative to their smaller counterparts.

As previously mentioned, the MES evaluates the ability of a dairy operation to trade reductions in milk output for reductions in emissions. We present the MES estimates by farm size in Table 6. In the early years, the estimates are highest for 
Table 3. Transient Technical Efficiency

\begin{tabular}{|c|c|c|c|c|}
\hline & & Medium Farms & Large Farms & \\
\hline Year & Small Farms & Percent & & Very Large Farms \\
\hline 1980 & 96.61 & 96.84 & 97.71 & 97.69 \\
\hline 1981 & 96.50 & 96.90 & 97.38 & 98.13 \\
\hline 1982 & 96.76 & 97.12 & 97.34 & 97.18 \\
\hline 1983 & 97.26 & 97.38 & 96.94 & 97.70 \\
\hline 1984 & 96.94 & 97.16 & 96.59 & 96.46 \\
\hline 1985 & 97.39 & 97.69 & 97.81 & 97.43 \\
\hline 1986 & 97.32 & 97.54 & 97.69 & 97.43 \\
\hline 1987 & 97.26 & 97.31 & 97.46 & 97.26 \\
\hline 1988 & 97.03 & 97.51 & 97.12 & 97.65 \\
\hline 1989 & 97.44 & 97.44 & 97.75 & 98.00 \\
\hline 1990 & 97.69 & 97.90 & 97.84 & 97.95 \\
\hline 1991 & 96.98 & 96.74 & 96.39 & 95.85 \\
\hline 1992 & 96.43 & 96.26 & 96.14 & 94.72 \\
\hline 1993 & 96.64 & 96.60 & 95.81 & 94.45 \\
\hline 1994 & 97.31 & 97.98 & 97.73 & 97.45 \\
\hline 1995 & 96.52 & 97.31 & 97.00 & 95.99 \\
\hline 1996 & 96.50 & 97.19 & 96.42 & 97.36 \\
\hline 1997 & 96.61 & 97.44 & 97.51 & 95.38 \\
\hline 1998 & 97.06 & 97.91 & 98.08 & 95.13 \\
\hline 1999 & 97.00 & 97.61 & 98.12 & 95.11 \\
\hline 2000 & 96.86 & 97.40 & 97.64 & 95.00 \\
\hline 2001 & 97.13 & 97.90 & 98.11 & 95.64 \\
\hline 2002 & 97.15 & 97.85 & 97.76 & 97.25 \\
\hline 2003 & 96.75 & 97.82 & 96.67 & 97.22 \\
\hline 2004 & 97.26 & 97.87 & 98.11 & 97.09 \\
\hline 2005 & 97.83 & 97.68 & 98.16 & 97.59 \\
\hline 2006 & 97.21 & 97.43 & 97.64 & 95.89 \\
\hline 2007 & 96.96 & 97.27 & 97.70 & 97.15 \\
\hline 2008 & 96.79 & 96.85 & 95.40 & 97.74 \\
\hline 2009 & 95.81 & 95.13 & 94.37 & 94.54 \\
\hline 2010 & 97.59 & 96.65 & 96.05 & 98.04 \\
\hline 2011 & 97.23 & 97.41 & 96.31 & 97.88 \\
\hline 2012 & 96.66 & 97.70 & 97.23 & 97.87 \\
\hline 2013 & 97.19 & 97.76 & 97.89 & 96.38 \\
\hline
\end{tabular}


Table 4. Persistent Technical Efficiency

\begin{tabular}{lcccc}
\hline & & Medium Farms & Large Farms & \\
Year & Small Farms & \multicolumn{2}{c}{ Percent } & Very Large \\
\hline $1980-2013$ & 96.32 & 96.75 & 96.26 & 95.25 \\
\hline
\end{tabular}

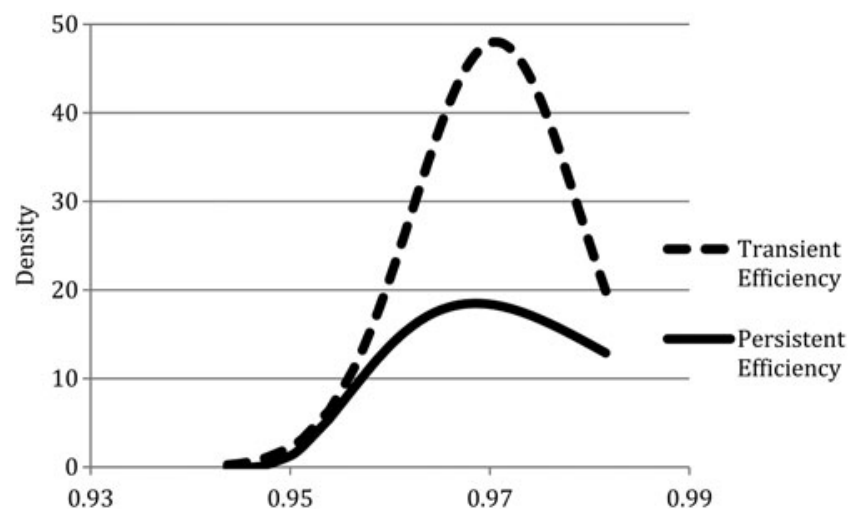

Figure 3. Kernel Density for Persistent (long-run) and Transient (short-run) Technical Efficiency

small dairy operations in absolute value. Once again, however, the situation changes in the mid-1990s, and the MES estimates of very large dairy farms begin to exceed those of other dairy operations. In addition, we find evidence that, in general, the MES values trend upward over time regardless of the size of the operations, which points to a reduction in the ability of dairies to make the substitution and curb their emissions in recent years.

\section{Concluding Remarks}

This study evaluates environmental efficiency for northeastern U.S. dairy farms of various sizes. Using data derived from Farm Credit East for 1980 through 2013, we estimate a directional output-distance function that measures joint production of milk and emission of GHGs while incorporating a four-way error approach that accounts for unobserved farm-size heterogeneity, timevarying transient technical efficiency, time-invariant persistent technical efficiency, and random errors. We also estimate shadow prices and output elasticities related to the cost of reducing emissions.

In terms of technical efficiency, we find that farm size has no significant effect. However, the computed shadow prices and output elasticities indicate that, 
Table 5. Shadow Prices

\begin{tabular}{|c|c|c|c|c|}
\hline \multirow{3}{*}{$\begin{array}{l}\text { Year } \\
1980\end{array}$} & \multirow{3}{*}{$\begin{array}{c}\text { Small Farms } \\
215.36\end{array}$} & \multirow{2}{*}{\multicolumn{2}{|c|}{$\begin{array}{c}\text { Medium Farms } \quad \text { Larg } \\
\text { Dollars per Ton }\end{array}$}} & \multirow{3}{*}{$\begin{array}{c}\text { Very Large Farms } \\
147.85\end{array}$} \\
\hline & & & & \\
\hline & & 192.01 & 145.31 & \\
\hline 1981 & 253.04 & 205.60 & 161.78 & 102.60 \\
\hline 1982 & 218.24 & 176.86 & 148.76 & 145.65 \\
\hline 1983 & 202.06 & 190.83 & 209.22 & 158.96 \\
\hline 1984 & 214.84 & 179.43 & 195.37 & 171.51 \\
\hline 1985 & 190.57 & 137.91 & 127.95 & 150.58 \\
\hline 1986 & 210.80 & 173.69 & 157.92 & 171.36 \\
\hline 1987 & 203.18 & 186.69 & 180.50 & 177.66 \\
\hline 1988 & 221.41 & 189.05 & 211.95 & 177.06 \\
\hline 1989 & 226.41 & 210.85 & 185.89 & 180.98 \\
\hline 1990 & 205.00 & 189.94 & 194.43 & 186.23 \\
\hline 1991 & 203.21 & 211.79 & 208.62 & 210.40 \\
\hline 1992 & 266.74 & 253.93 & 247.23 & 265.18 \\
\hline 1993 & 234.72 & 222.38 & 244.06 & 260.31 \\
\hline 1994 & 185.46 & 137.60 & 171.60 & 192.17 \\
\hline 1995 & 212.22 & 172.88 & 184.42 & 210.36 \\
\hline 1996 & 239.55 & 212.18 & 225.57 & 221.66 \\
\hline 1997 & 181.78 & 163.08 & 180.16 & 192.99 \\
\hline 1998 & 192.00 & 170.62 & 170.10 & 216.69 \\
\hline 1999 & 170.02 & 167.26 & 169.26 & 196.83 \\
\hline 2000 & 148.11 & 156.12 & 157.72 & 179.55 \\
\hline 2001 & 168.56 & 158.22 & 176.30 & 205.72 \\
\hline 2002 & 142.27 & 133.77 & 158.81 & 179.08 \\
\hline 2003 & 161.66 & 135.40 & 183.37 & 172.49 \\
\hline 2004 & 207.28 & 178.85 & 192.52 & 239.28 \\
\hline 2005 & 170.50 & 158.31 & 178.22 & 221.22 \\
\hline 2006 & 176.38 & 161.54 & 173.38 & 214.22 \\
\hline 2007 & 267.32 & 244.35 & 245.29 & 312.70 \\
\hline 2008 & 280.83 & 261.91 & 293.63 & 314.74 \\
\hline 2009 & 200.98 & 195.91 & 219.78 & 235.92 \\
\hline 2010 & 200.43 & 222.36 & 237.99 & 276.54 \\
\hline 2011 & 219.76 & 210.76 & 256.10 & 294.61 \\
\hline 2012 & 210.59 & 195.94 & 223.50 & 241.86 \\
\hline 2013 & 203.63 & 206.48 & 219.72 & 250.50 \\
\hline Average & 199.13 & & & \\
\hline Maximum & 314.74 & & & \\
\hline Minimum & 102.60 & & & \\
\hline
\end{tabular}


Table 6. Morishima Elasticity of Substitution

\begin{tabular}{|c|c|c|c|c|}
\hline Year & Small Farms & Medium Farms & Large Farms & Very Large Farms \\
\hline 1980 & -1.72 & -1.70 & -1.64 & -1.65 \\
\hline 1981 & -1.79 & -1.73 & -1.67 & -1.60 \\
\hline 1982 & -1.76 & -1.70 & -1.67 & -1.67 \\
\hline 1983 & -1.74 & -1.73 & -1.77 & -1.69 \\
\hline 1984 & -1.78 & -1.73 & -1.76 & -1.73 \\
\hline 1985 & -1.77 & -1.68 & -1.67 & -1.71 \\
\hline 1986 & -1.83 & -1.76 & -1.74 & -1.77 \\
\hline 1987 & -1.82 & -1.79 & -1.79 & -1.79 \\
\hline 1988 & -1.88 & -1.82 & -1.88 & -1.80 \\
\hline 1989 & -1.87 & -1.84 & -1.79 & -1.79 \\
\hline 1990 & -1.82 & -1.80 & -1.81 & -1.80 \\
\hline 1991 & -1.92 & -1.97 & -1.96 & -1.98 \\
\hline 1992 & -2.10 & -2.08 & -2.07 & -2.15 \\
\hline 1993 & -2.05 & -2.03 & -2.12 & -2.21 \\
\hline 1994 & -1.89 & -1.77 & -1.87 & -1.94 \\
\hline 1995 & -2.02 & -1.90 & -1.95 & -2.06 \\
\hline 1996 & -2.05 & -1.97 & -2.03 & -2.02 \\
\hline 1997 & -1.93 & -1.88 & -1.94 & -1.99 \\
\hline 1998 & -1.89 & -1.84 & -1.85 & -1.99 \\
\hline 1999 & -1.86 & -1.86 & -1.88 & -1.97 \\
\hline 2000 & -1.87 & -1.91 & -1.92 & -2.01 \\
\hline 2001 & -1.86 & -1.83 & -1.90 & -2.00 \\
\hline 2002 & -1.90 & -1.87 & -1.99 & -2.09 \\
\hline 2003 & -1.98 & -1.88 & -2.10 & -2.06 \\
\hline 2004 & -1.99 & -1.91 & -1.96 & -2.15 \\
\hline 2005 & -1.92 & -1.88 & -1.96 & -2.15 \\
\hline 2006 & -2.08 & -2.02 & -2.09 & -2.34 \\
\hline 2007 & -2.12 & -2.06 & -2.07 & -2.36 \\
\hline 2008 & -2.28 & -2.21 & -2.38 & -2.51 \\
\hline 2009 & -2.29 & -2.28 & -2.48 & -2.64 \\
\hline 2010 & -2.04 & -2.15 & -2.24 & -2.49 \\
\hline 2011 & -1.99 & -1.97 & -2.14 & -2.32 \\
\hline 2012 & -2.04 & -2.00 & -2.12 & -2.21 \\
\hline 2013 & -1.97 & -1.99 & -2.05 & -2.18 \\
\hline Average & -1.95 & & & \\
\hline Minimum & -1.60 & & & \\
\hline Maximum & -2.64 & & & \\
\hline
\end{tabular}


beginning in the mid-1990s, very large dairy operations faced higher shadow prices and MES values relative to other farms, and in later years, we find that the output elasticities generally trended up for all farm sizes.

As noted, the dairy industry both nationwide and in the Northeast has been consolidating (USDA 2014, Winsten et al. 2010). Among the benefits of larger dairy operations are economies of scale, increased productivity (Mosheim and Lovell 2009), and a higher cash margin per unit of milk sold (MacDonald, McBride, and O'Donoghue 2007). In recent years, large dairy operations in the Northeast have been characterized by confinement of large herds in relatively small and restricted areas. These confinement-feeding systems have been adopted to reduce production costs and raise the output of milk per cow (Winsten et al. 2010). However, having large herds in a confined area contributes to higher levels of emissions since the cows are kept and raised in restricted lots rather than on rangeland and pasture.

According to Farm Credit East, 65 of the 517 farms surveyed in 2013 maintained more than 700 cows. Dairy operations of that size are required to meet statutory environmental standards under the Clean Water Act of 1972 and rules regarding concentrated animal feeding operations (EPA 2008) that seek to protect the quality of ground water and public health. As a result, these large dairy farms would have been required to obtain manure discharge permits and to develop nutrient management plans to minimize the negative effects of animal waste (Tao et al. 2012). These requirements may have raised the cost of manure management and, hence, shadow prices, and the difficulty associated with reducing emissions while maintaining or raising the output of milk would then be reflected in higher MES values.

Our results suggest that any policy action taken to curb emissions could adversely affect the competitiveness of large-scale dairy operations. This poses a significant dilemma for policymakers given the importance of dairy farming to local economies and preservation of agricultural landscapes in the Northeast (Johnston 2002). We propose that public policy interventions be directed toward large dairy operations to assist them in improving their environmental efficiency and to smaller dairy farms to assist them in becoming environmentally sustainable as they expand. These types of assistance could take various forms, including an emphasis on extension services that could provide expert advice regarding ways to reduce pollution and direct subsidies and tax breaks for farms that install pollution-reduction equipment such as anaerobic digesters.

\section{References}

Ahmad, M., and B.E. Bravo-Ureta. 1996. “Technical Efficiency Measures for Dairy Farms Using Panel Data: A Comparison of Alternative Model Specifications." Journal of Productivity Analysis 7(4): 399-415.

Atkinson, S.E., and J.H. Dorfman. 2005. "Bayesian Measurement of Productivity and Efficiency in the Presence of Undesirable Outputs: Crediting Electric Utilities for Reducing Air Pollution." Journal of Econometrics 126(2): 445-468. 
Batie, S. 2003. "The Multifunctional Attributes of Northeastern Agriculture: A Research Agenda." Agricultural and Resource Economics Review 32(1): 1-8.

Blackorby, C., and R.R. Russell. 1989. "Will the Real Elasticity of Substitution Please Stand Up?" American Economic Review 79(4): 882-888.

Bravo-Ureta, B.E., D. Solis, V.H. Moreira, J.F. Maripani, A. Thiam, and T. Rivas. 2007. "Technical Efficiency in Farming: A Meta-regression Analysis." Journal of Productivity Analysis 27(1): 57-72.

Chambers, R.G., Y. Chung, and R. Färe. 1996. "Benefit and Distance Functions." Journal of Economic Theory 70(2): 407-419.

— Y. Chung, and R. Färe. 1998. "Profit, Directional Distance Functions, and Nerlovian Efficiency." Journal of Optimization Theory and Applications 98(2): 351-364.

Chung, Y.H., R. Färe, and S. Grosskopf. 1997. "Productivity and Undesirable Outputs: A Directional Distance Function Approach." Journal of Environmental Management 51(3): 229-240.

Colombi, R., S.C. Kumbhakar, G. Martini, and G. Vittadini. 2014. "Closed-skew Normality in Stochastic Frontiers with Individual Effects and Long/Short-run Efficiency." Journal of Productivity Analysis 42(2): 123-136.

Cuesta, R.A., C.A.K. Lovell, and J. Zofio. 2009. "Environmental Efficiency Measurement with Translog Distance Functions: A Parametric Approach." Ecological Economics 68(8): 2232-2242.

Environmental Protection Agency. 2008. "Revised National Pollutant Discharge Elimination System Permit Regulation and Effluent Limitations Guidelines for Concentrated Animal Feeding Operations; Final Rule." Federal Register 73(225): 70418-70486.

- 2009. "Mandatory Reporting of Greenhouse Gases; Final Rule." Federal Register 74 (209): 56337-56489.

- 2011. "Inventory of U.S. Greenhouse Gas Emissions and Sinks: 1990-2009." 430-R-11005, Environmental Protection Agency, Washington, DC.

—. 2012a. "U.S. Greenhouse Gas Emissions and Sinks: 1990-2010." Annex 2, 430-R-12001, Environmental Protection Agency, Washington, DC.

—. 2012b. "Greenhouse Gas Equivalencies Calculator" web page, www.epa.gov/ cleanenergy/energy-resources/calculator.html (accessed August 21, 2014). Environmental Protection Agency, Washington, DC.

Färe, R. 2010. "Directional Distance Functions and Public Transportation: A Comment." Transportation Research 15(2): 105-109.

- S. Grosskopf, D. Noh, and W. Weber. 2005. "Characteristics of a Polluting Technology: Theory and Practice." Journal of Econometrics 126(2): 469-492.

- and D. Primont. 1995. Multi-output Production and Duality: Theory and Applications. Boston, MA: Kluwer Academic Publishers.

Fernandez, C., G. Koop, and M.F.J. Steel. 2002. "Multiple-output Production with Undesirable Outputs: An Application to Nitrogen Surplus in Agriculture." Journal of the American Statistical Association 97(458): 432-442.

Filippini, M., and W. Greene. 2014. "Persistent and Transient Productive Inefficiency: A Maximum Simulated Likelihood Approach." Working Paper 14/197, Center for Economic Research, ETH Zurich.

Gerber, P.J., H. Steinfeld, B. Henderson, A. Mottet, C. Opio, J. Dijkman, A. Falcucci, and G. Tempio. 2013. "Tackling Climate Change through Livestock: A Global Assessment of Emissions and Mitigation Opportunities." Food and Agriculture Organization, United Nations, Rome.

Johnston, R.J. 2002. "Conserving Farm and Forest in a Changing Rural Landscape: Current and Potential Contributions of Economics." Regional Rural Development Paper 11, Northeast Regional Center for Rural Development, Pennsylvania State University, University Park.

Kellerman, M.A. 2015. "Total Factor Decomposition and Unobserved Heterogeneity in Stochastic Frontier Models." Agricultural and Resource Economics Review 44(1): 124-148. 
Key, N., and S. Sneeringer. 2014. "Potential Effects of Climate Change on the Productivity of U.S. Dairies." American Journal of Agricultural Economics 96(4): 1136-1156.

Kumbhakar, S.C., S. Ghosh, and J.T. McGuckin. 1991. "A Generalized Production Frontier Approach for Estimating Determinants of Inefficiency in U.S. Dairy Farms." Journal of Business and Economic Statistics 9(3): 279-286.

—, G. Lien, and J.B. Hardaker. 2014. "Technical Efficiency in Competing Panel Data Models: A Study of Norwegian Grain Farming." Journal of Productivity Analysis 41(2): 321-337.

—, E.G. Tsionas, and T. Sipilainen. 2009. "Joint Estimation of Technology Choice and Technical Efficiency: An Application to Organic and Conventional Dairy Farming." Journal of Productivity Analysis 31(3): 151-161.

Kuosmanen, T. 2005. "Weak Disposability in Nonparametric Production Analysis with Undesirable Outputs." American Journal of Agricultural Economics 87(4): 1077-1082.

MacDonald, J.M., W.D. McBride, and E.J. O’Donoghue. 2007. “Low Costs Drive Production to Large Dairy Farms.” Amber Waves 5(4): 30-35.

Mosheim, R., and C.A.K. Lovell. 2009. "Scale Economies and Inefficiency of U.S. Dairy Farms." American Journal of Agricultural Economics 91(3): 777-794.

Mosier, A.R. 1994. "Nitrous Oxide Emissions from Agricultural Soils." Fertilizer Research 37(3): 191-200.

Mukherjee, D., B.E. Bravo-Ureta, and A. De Vries. 2013. "Dairy Productivity and Climatic Conditions: Econometric Evidence from Southeastern United States." Australian Journal of Agricultural and Resource Economics 57(1): 123-140.

Njuki, E., and B.E. Bravo-Ureta. 2015. "The Economic Costs of Environmental Regulation in U.S. Dairy Farming: A Directional Distance Function Approach." American Journal of Agricultural Economics 97(4): 1087-1106.

O’Donnell, C.J. 2007. "Estimating the Characteristics of Polluting Technologies." Selected paper presented at the 51st Annual Conference of the Australian Agricultural and Resource Economics Society, Queenstown, New Zealand.

- 2016. "Using Information about Technologies, Markets, and Firm Behavior to Decompose a Proper Productivity Index." Journal of Econometrics 190(2): 328-340.

Picazo-Tadeo, A.J., E. Reig-Martinez, and F. Hernandez-Sancho. 2005. "Directional Distance Functions and Environmental Regulation." Resource and Energy Economics 27(2): 131142.

Qi, L., B.E. Bravo-Ureta, and V.E. Cabrera. 2015. "From Cold to Hot: Climatic Effects and Productivity in Wisconsin Dairy Farms." Journal of Dairy Science 98(12): 8664-8677.

Reinhard, S., C.A.K. Lovell, and G. Thijssen. 1999. "Econometric Estimation of Technical and Environmental Efficiency: An Application to Dutch Dairy Farms." American Journal of Agricultural Economics 81(1): 44-60.

Shephard, R.W. 1970. Theory of Costs and Production Functions. Princeton, NJ: Princeton University Press.

— 69-90.

Solow, R. 1957. "Technical Change and the Aggregate Production Function." Review of Economics and Statistics 39(3): 312-320.

Tao, H., T.F. Morris, B. Bravo-Ureta, R. Meinart, and J. Neafsey. 2012. "Nutrient Applications Reported by Farmers Compared with Performance-based Nutrient Management Plans." Agronomy Journal 104(2): 437-447.

Thoma, G., J. Popp, D. Nutter, D. Shonnard, R. Ulrich, M. Matlock, D.S. Kim, Z. Neiderman, N. Kemper, C. East, and F. Adom. 2013. "Greenhouse Gas Emissions from Milk Production and Consumption in the United States: A Cradle-to-grave Life Cycle Assessment circa 2008." International Dairy Journal 31(1): S3-S14.

Tietenberg, T.H. 2006. "Emissions Trading: Principles and Practice." Resources for the Future, Washington, DC. 
Tsionas, E.G., and S.C. Kumbhakar. 2014. "Firm Heterogeneity, Persistent and Transient Technical Inefficiency: A Generalized True Random-Effects Model." Journal of Applied Econometrics 29(1): 110-132.

U.S. Congress. 1990. Clean Air Act Amendments of 1990. Pub. L. No. 101-549, 104 Stat. 2399.

U.S. Department of Agriculture. 2010. "Overview of the United States Dairy Industry." Agricultural Statistics Board, National Agricultural Statistics Service, Washington, DC.

- 2014. "Milk Production, Disposition, and Income: 2013 Summary." National Agricultural Statistics Service, USDA, Washington, DC.

U.S. Supreme Court. 2007. Commonwealth of Massachusetts et al. vs. Environmental Protection Agency et al. Docket number 05-1120, decided April 2, 2007.

Winsten, J.R., C.D. Kerchner, A. Richardson, A. Lichau, and J.M. Hyman. 2010. "Trends in the Northeast Dairy Industry: Large-scale Modern Confinement Feeding and Managementintensive Grazing." Journal of Dairy Science 93(4): 1759-1769. 